Check for updates

Cite this: Chem. Commun., 2021, 57,12972

Received 7th October 2021,

Accepted 12th November 2021

DOI: $10.1039 / \mathrm{d} 1 \mathrm{cc} 05671 \mathrm{a}$

rsc.li/chemcomm

\section{Effect of tert-butyl groups on electronic communication between redox units in tetrathiafulvalene-tetraazapyrene triads $\dagger$}

\author{
Ping Zhou, ${ }^{a}$ Ulrich Aschauer, (D) a Silvio Decurtins, ${ }^{a}$ Thomas Feurer, ${ }^{b}$ \\ Robert Häner (iD ${ }^{a}$ and Shi-Xia Liu (D) *a
}

The electronic effect of tert-butyl groups on intramolecular throughbond interactions between redox units in tetrathiafulvalene-tetraazapyrene (TAP) triads is investigated. The insertion of tert-butyl groups raises the TAP-localised LUMO level by $0.21 \mathrm{eV}$, in fairly good agreement with $0.17 \mathrm{eV}$ determined by DFT calculations.

Tetraazapyrene (TAP), a prototype of nitrogenated polycyclic aromatic hydrocarbons (N-PAHs), exhibits intrinsic optoelectronic and electrochemical properties as well as high thermal and chemical stability, which are important requirements for its diverse applications in the field of organic (opto)electronics. ${ }^{1,2}$ However, reports on synthetic approaches to the TAP scaffold and its derivatization are quite limited in the literature. ${ }^{3}$ In 2012, a series of 2,7-substituted TAP derivatives was prepared and tested as n-type semiconductors in organic field-effect transistors (OFETs). ${ }^{1}$ It has been demonstrated that the electronic properties are significantly affected by the nature of substituents at the core positions. Inspired by these appealing results, we have embarked on the design and synthesis of tetrathiafulvalene (TTF)functionalized TAPs to create triads (Scheme 1) with promising material properties.

Within the context of organic conductors, TTF acts as a strong $\pi$-electron donor and takes a prominent place in this class of materials. ${ }^{4}$ By virtue of its unique redox properties, it has been incorporated into a variety of (supra)molecular architectures for the development of highly efficient (opto)electronic materials. ${ }^{5}$ Among them, TTF-based electron donor-acceptor (D-A) ensembles have attracted a lot of attention with a special focus on redox modulation of intramolecular charge transfer (ICT) and chemical/optical control over multiple ICT pathways. ${ }^{6,7}$ Herein, we report a facile and efficient synthetic protocol to 2,7-di-tert-butyl-1,3,6,8-tetraazapyrene (2) as well as the further

\footnotetext{
${ }^{a}$ Department of Chemistry, Biochemistry and Pharmaceutical Sciences, University of Bern, Freiestrasse 3, Bern CH-3012, Switzerland. E-mail: shi-xia.liu@unibe.ch

${ }^{b}$ Institute of Applied Physics, University of Bern, Sidlerstrasse 5, Bern CH-3012, Switzerland

$\dagger$ Electronic supplementary information (ESI) available. See DOI: 10.1039/d1cc05671a
}

derivatization by 4 -fold core-bromination for subsequent nucleophilic substitutions with a cyanoethyl-protected TTF (4), leading to the formation of two triads TTF-TAP and TTF- $t$-Bu-TAP with a D-A-D architecture (Scheme 1).

It is well known that bulky tert-butyl groups have been widely incorporated into large N-PAH molecules for improved solubility because of suppressed aggregation. ${ }^{8,9}$ Also, tert-butylation has been exploited to bias regioselectivity, e.g. insertion of tert-butyl groups into 2,7 positions of pyrene can avoid the reaction occurring at the electron-rich 1,3,6,8-carbons instead of the $K$-region $\left(4,5,9,10\right.$-carbons). ${ }^{10}$ This work aims to reveal the effects of tertbutyl groups ${ }^{9,11}$ on the electronic characteristics of the triads. Redox and optical properties of TTF-TAP and TTF- $t$-Bu-TAP have been investigated in detail. The insertion of tert-butyl groups at positions 2 and 7 of the TAP core has indeed a very pronounced effect on the TAP-localised LUMO energy level.

As illustrated in Scheme 1, our synthetic approach to the 2,7substituted TAP scaffold 2 involves a twofold cyclocondensation of naphthalene-1,4,5,8-tetraamine tin(II) salt (1) ${ }^{12}$ with an excess of pivalic anhydride, followed by oxidation of the intermediate 1,8dihydropyrimido[ $[4,5,6-g h]$ perimidine. This reaction was performed at reflux in THF in the presence of catalytic amounts of triethylamine, affording 2 as a pale-yellow powder in a yield of $60 \%$. The subsequent core-bromination was accomplished to yield $3(70 \%)$ in concentrated sulfuric acid in the presence of $\mathrm{Br}_{2}$ with catalytic amounts of iodine, similar to the synthetic procedure reported for 5. ${ }^{1}$ Both 4 -fold core-brominated compounds $\mathbf{3}$ and $\mathbf{5}$ undergo nucleophilic substitution with 4 in the presence of $\mathrm{CsOH} / \mathrm{MeOH}$ to produce the desired products TTF- $\boldsymbol{t}$-Bu-TAP and TTF-TAP in a yield of $18 \%$ and $24 \%$, respectively. These as-prepared compounds were unambiguously characterized by ${ }^{1} \mathrm{H}$ and ${ }^{13} \mathrm{C}$ NMR, cyclic voltammetry, UV-Vis-NIR spectroscopy and high-resolution mass spectrometry. All analytical data match well with their chemical structures.

The electrochemical properties of TTF-TAP, TTF- $t$-Bu-TAP and the reference compounds $\mathbf{2}$ and $\mathbf{4}$ were investigated by cyclic voltammetry and differential pulse voltammetry in $\mathrm{CH}_{2} \mathrm{Cl}_{2}$ (Fig. 1 and Table S1, Fig. S1, ESI $\dagger$ ). TTF-TAP undergoes three distinct 


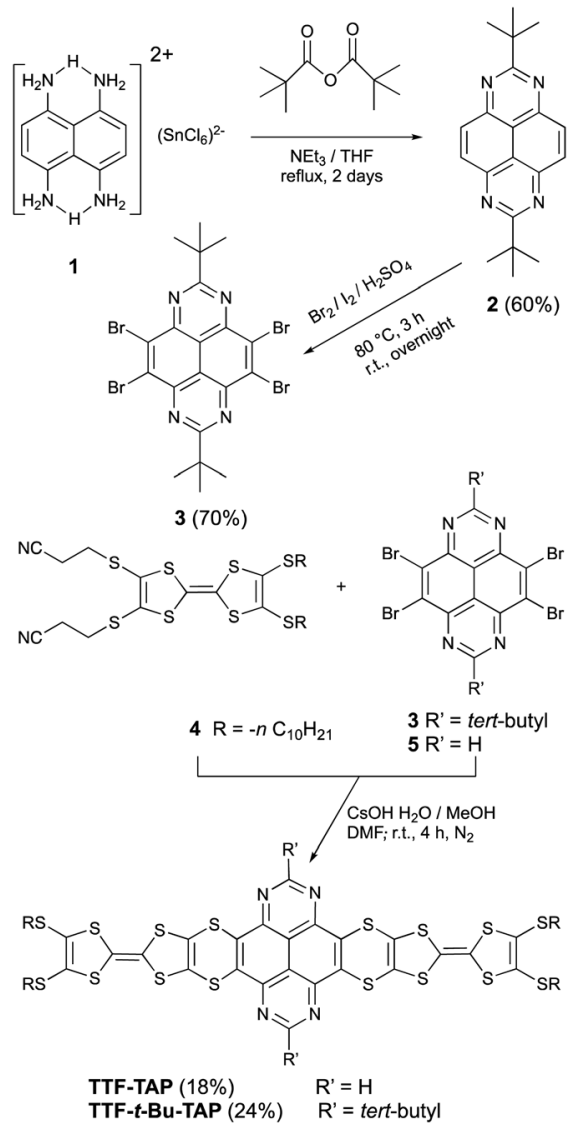

Scheme 1 Synthesis of a key precursor 3, triads TTF-TAP and TTF-t-Bu-TAP.
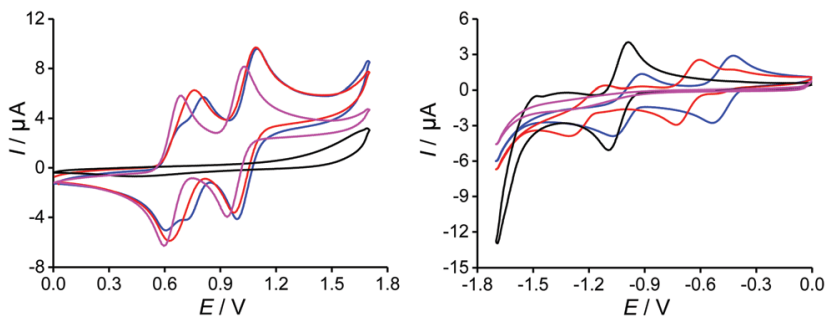

Fig. 1 Cyclic voltammograms of TTF-TAP (blue), TTF-t-Bu-TAP (red), $t$ Bu-TAP 2 (black) and TTF precursor 4 (pink) were measured in dichloromethane solution $\left(c=3 \times 10^{-4} \mathrm{M}\right.$ ), containing $0.1 \mathrm{M} \mathrm{TBAPF}_{6}$ as the supporting electrolyte at room temperature, Pt working electrode, Ag/ $\mathrm{AgCl}$ electrode as the reference electrode and the scan rate at $100 \mathrm{mV} \mathrm{s}^{-1}$.

reversible oxidation processes at $0.63 \mathrm{~V}, 0.77 \mathrm{~V}$ and at $1.06 \mathrm{~V}$ (vs. Ag/AgCl), indicating that two TTF units are successively oxidized to TTF radical cation species and simultaneously to TTF dication species. This phenomenon remains even in a very diluted solution (Fig. S2, ESI $\dagger$ ), indicating that the possibility of associations between TTF-TAP molecules is ruled out, which can further be verified by concentration-dependent UV-Vis spectra (Fig. S3, ESI $\dagger$ ). The occurrence of step-wise oxidation processes for the generation of the radical cation species points to significant intramolecular through-bond interactions between two TTF units, as previously observed in analogous molecules. ${ }^{13}$ In the negative potential window, two reversible reduction waves at $-0.46 \mathrm{~V}$ and
$-0.98 \mathrm{~V}$ are observed, corresponding to the sequential addition of electrons to the TAP unit. Interestingly, the incorporation of tertbutyl group has an appreciable influence on the redox properties of TTF and TAP units. TTF-t-Bu-TAP shows only two reversible oxidation waves at $0.70 \mathrm{~V}$ and $1.03 \mathrm{~V}$ (Fig. S1, ESI $\dagger$ ), suggesting that two TTF units are simultaneously oxidized to their radical cation and dication species, respectively. Similarly, it undergoes two reversible reductions at $-0.67 \mathrm{~V}$ and $-1.19 \mathrm{~V}$, which, however, are negatively shifted compared to TTF-TAP. Consequently, the insertion of tert-butyl groups raises the LUMO level by $0.21 \mathrm{eV}$, very probably due to the hyperconjugation effect of the tert-butyl groups, but an electrostatic destabilization of the TAP-localized $\pi^{*}$ MO can also be invoked based on computed electrostatic potentials at nuclear positions (Table S2, ESI $\dagger$ ). The first reduction potential values of both triads are positively shifted with respect to that of the TAP, ref. 2 indicative of the extended conjugation via four $S$ atoms caused by the covalent linkage of two TTF units to the central TAP core. In stark contrast, their oxidation potentials are either shifted only slightly positively or remain almost unchanged compared to those of the TTF precursor 4, most likely because of the lack of an effective electronic communication between the redox units. The latter is a consequence of nonplanar conformations of the triads, as shown by the corresponding calculated structures (Fig. S4, ESI $\dagger$ ). As a result, both triads show pronouncedly lowered LUMO energies while maintaining similar HOMO energies with respect to the constituting moieties 2 and 4, which matches well the UV-Vis spectroscopic results presented below.

TTF-TAP and TTF- $t$-Bu-TAP strongly absorb in the UVVisible-NIR spectral region as evidenced by their yellow-green colour. Their absorption spectra in $\mathrm{CH}_{2} \mathrm{Cl}_{2}$ are depicted in Fig. 2 .

In the UV and blue part of the optical spectra, the observed strong absorption bands are attributed to $\pi-\pi^{*}$ transitions localized on the TTF and TAP cores, similarly to the reference compounds 2 and 4, only with bathochromic shifts owing to extended conjugation. By comparison of TTF-t-Bu-TAP (red) to TTF-TAP (blue), the insertion of the tert-butyl groups leads to

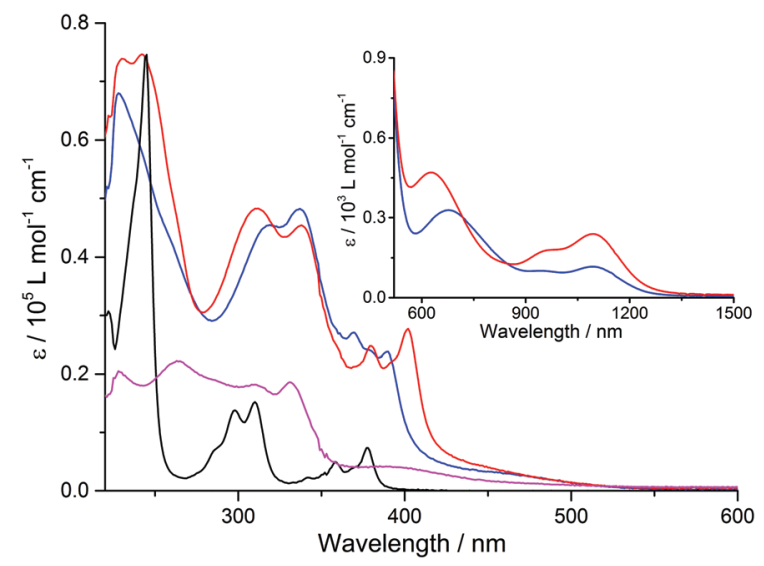

Fig. 2 UV-Vis absorption spectra of TTF-TAP (blue), TTF-t-Bu-TAP (red), $t$-Bu-TAP 2 (black) and TTF precursor 4 (pink) $\left(c=7 \times 10^{-6} \mathrm{M}\right)$ in $\mathrm{CH}_{2} \mathrm{Cl}_{2}$ at r.t. Inset: Vis-NIR absorption spectra of TTF-TAP, TTF-t-Bu-TAP (c $=7 \times 10^{-4} \mathrm{M}$ ) in $\mathrm{CH}_{2} \mathrm{Cl}_{2}$ at r.t. 
noticeable bathochromic shifts of the absorption bands in a range of $360 \mathrm{~nm}$ to $450 \mathrm{~nm}$ and a slight hypsochromic shift of the absorption band around $320 \mathrm{~nm}$. As these transitions correspond mainly to $\pi-\pi^{*}$ excitations of the TAP core, the observed shifts can be accounted for by the fact that the presence of bulky tert-butyl groups at 2,7-positions of the TAP is unfavourable for possible $H$ - and $J$-aggregation and impedes effective intermolecular interactions. More importantly, both triads exhibit two weak and broad absorption bands centered at $680 \mathrm{~nm}$ and $1100 \mathrm{~nm}$ for TTF-TAP, and $630 \mathrm{~nm}$ and $1100 \mathrm{~nm}$ for TTF- $\boldsymbol{t}$-Bu-TAP, which are not observed, however, in solutions of the acceptor 2 and donor 4 components. Consequently, these absorptions reflect ICT transitions dominated by excitations from MOs localized on the TTF units to the LUMO localized on the TAP core. These assignments are corroborated by the computational results (see below). An attempt can be made to approach with some uncertainties the onsets of the very weak and broad absorption bands of both triads in the NIR region (Fig. 2) while extrapolating the linear portions on their red sides to the abscissa. Their optical bandgaps lie approximately between $1 \mathrm{eV}$ and $0.92 \mathrm{eV}$, with the higher value assigned to the more steeply falling absorption edge of the TTF-t-Bu-TAP triad, a trend compliant with the electrochemical study.

In order to shed more light on the intramolecular electronic interactions between the TTF and TAP units in TTF-TAP and TTF- $\boldsymbol{t}$-Bu-TAP, spectroelectrochemical measurements were carried out. Chemical oxidation of TTF-TAP and TTF- $\boldsymbol{t}$-Bu-TAP was performed by successive addition of $\mathrm{NOSbF}_{6}$ aliquots at room temperature. As shown in Fig. 3 for TTF-TAP, a progressive reduction of the absorbance of the high energy $\pi-\pi^{*}$ transitions is accompanied with a concomitant appearance of new absorption bands at $452 \mathrm{~nm}$ and $835 \mathrm{~nm}$ which reach their maximum values upon addition of 12 equiv. of $\mathrm{NOSbF}_{6}$. The lowest energy absorption band is continuously blue-shifted during the oxidation process. These new transitions are characteristic of the newly formed cationic $\mathrm{TTF}^{\bullet+}$ radical within a D-A-D triad. ${ }^{13,14}$ In contrast, the initial oxidation of TTF-t-Bu-TAP leads to a broad absorption band which emerges at $905 \mathrm{~nm}$ and, after

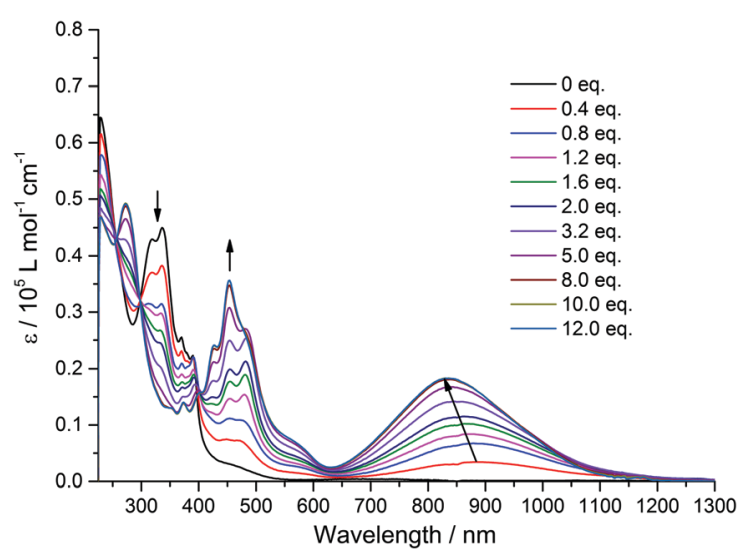

Fig. 3 Variation of UV-Vis-NIR absorption spectra of TTF-TAP $\left(7 \times 10^{-6} \mathrm{M}\right)$ in $\mathrm{CH}_{2} \mathrm{Cl}_{2}$ upon successive addition of aliquots of $\mathrm{NOSbF}_{6}$ at room temperature.

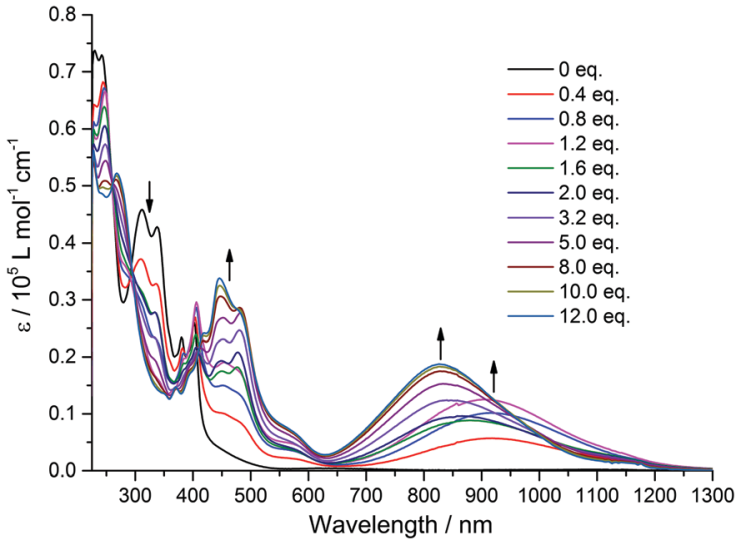

Fig. 4 Variation of UV-Vis-NIR absorption spectra of TTF- $t$-Bu-TAP $\left(7 \times 10^{-6} \mathrm{M}\right)$ in $\mathrm{CH}_{2} \mathrm{Cl}_{2}$ upon successive addition of aliquots of $\mathrm{NOSbF}_{6}$ at room temperature.

addition of 1.2 equiv., suddenly moves towards higher energy $(830 \mathrm{~nm})$ with increasing amounts of $\mathrm{NOSbF}_{6}$ (Fig. 4). Again, it reaches its maximum value upon addition of 12 equiv. of $\mathrm{NOSbF}_{6}$. In both cases, this broad and distinctly asymmetric absorption band in the NIR region is mainly attributed to electronic transitions localized on the $\mathrm{TTF}^{\bullet^{+}}$unit with some back ICT character from the TAP unit to the radical species $\mathrm{TTF}^{\bullet+}$, however to a much lesser extent than previously reported in TTF-fused D-A systems. ${ }^{7,14,15}$ This result could be accounted for by their non-planar geometries (Fig. S4, ESI $\dagger$ ). Moreover, the observed variance in the evolution of the absorption spectra in this spectral region is very probably due to different degrees of intermolecular $\pi$-dimerization of the $\mathrm{TTF}^{\bullet+}$ radical cation imparted by sequential or simultaneous oxidation of two TTF cores in TTF-TAP and TTF-t-Bu-TAP, as corroborated by CV measurements.

To characterize and verify the various electronic transitions, TD-DFT calculations were performed using the Gaussian 16 package at the B3LYP/6-31G(d,p) level of theory. ${ }^{16}$ The predicted absorption spectra of TTF-TAP and TTF-t-Bu-TAP (Fig. S5, ESI $\dagger$ ) are in fairly good agreement with experimental results. Fig. 5 illustrates the frontier MOs of TTF-TAP which are involved in the ICT transitions. The HOMO and HOMO-1

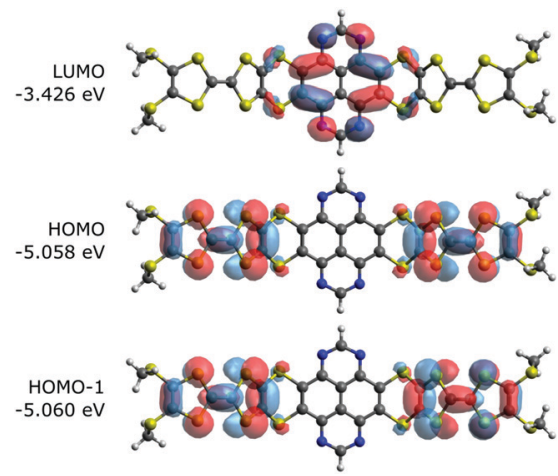

Fig. 5 Frontier molecular orbitals of TTF-TAP that are involved in the ICT transition. 
represent the symmetric and asymmetric combinations of the two donor TTF MOs. Analogously, HOMO-2 and HOMO-3 combine both pending units, however, the TAP core is now partly involved in their electron densities (Fig. S6, ESI $\dagger$ ). The LUMO is localized at the acceptor TAP core with some extension to the adjacent sulfur atoms. The frontier MOs of TTF-t-Bu-TAP (Fig. S6, ESI $\dagger$ ) show the same characteristics. The calculated HOMO-LUMO gap amounts to $1.63 \mathrm{eV}$ for TTF-TAP and $1.76 \mathrm{eV}$ for TTF- $\boldsymbol{t}$-Bu-TAP.

The predicted absorption spectra for both triads (Fig. S5, ESI $\dagger$ ) show a manifold of $\pi-\pi^{*}$ transitions with high oscillator strengths at wavelength below $450 \mathrm{~nm}$, in good agreement with the measured absorption spectra. The measured low intensity absorptions around 630-680 $\mathrm{nm}$ are well characterized by calculated HOMO-2/HOMO-3 $\rightarrow$ LUMO excitations, fairly close to that energy range and also with low oscillator strengths (Tables S3 and S4, ESI $\dagger$ ). The same holds for the very weak longest wavelength absorptions which are now attributed to HOMO/HOMO-1 $\rightarrow$ LUMO excitations. Clearly, the orbital overlap among these MOs is little, which leads to a small transition dipole moment, hence low intensities for their transitions. To note, there is a distinct blue-shift for these calculated excitations for the TTF-t-Bu-TAP triad compared to TTF-TAP. Fig. S7 and Tables S5-S8 (ESI $\dagger$ ) show the calculated transitions and the involved open-shell molecular orbitals (Fig. S8-S11, ESI $\dagger$ ) for the +1 and +2 cationic states of both triads which by and large look similar. Upon oxidation of two TTF units to their radical states, the involved molecular orbitals of the NIR transitions reveal that the majority of charge remains on the TTF units.

In conclusion, the newly synthesized TTF-TAP and TTF-t-BuTAP absorb in the UV-Vis-NIR spectral region due to ICT excitations from TTF units to the central TAP core. Interestingly, the two TTF units of TTF-TAP are sequentially oxidized to the TTF radical cation species while this process occurs simultaneously in TTF-tBu-TAP. With respect to TTF-TAP, the LUMO level of TTF- $t$-Bu-TAP is raised by $0.21 \mathrm{eV}$. Also it is worthy of note that a modification at the TTF side would potentially be a tuning factor in the molecule as well. ${ }^{17}$ Our findings represent a novel concept in the manipulation of the relative HOMO and LUMO energetic positions of organic donor-acceptor ensembles by chemical modification.

We greatly appreciate preliminary clarifications on these systems by Dirk Guldi. This work was financially supported by the Swiss NSF through the NCCR MUST "Molecular Ultrafast Science and Technology", the Swiss NSF (200020_188468 and 200021_204053), and the Swiss NSF professorship grants (PP00P2_157615 and PP00P2_187185). Computations were performed on Ubelix, the HPC cluster at the University of Bern.

\section{Conflicts of interest}

There are no conflicts to declare.

\section{Notes and references}

1 S. Geib, S. C. Martens, U. Zschieschang, F. Lombeck, H. Wadepohl, H. Klauk and L. H. Gade, J. Org. Chem., 2012, 77, 6107.
2 S. C. Martens, L. Hahn, F. Lombeck, A. Rybina, H. Wadepohl and L. H. Gade, Eur. J. Org. Chem., 2013, 5295; M. J. Sienkowska, J. M. Farrar, F. Zhang, S. Kusuma, P. A. Heiney and P. Kaszynski, J. Mater. Chem., 2007, 17, 1399; Y.-R. Shi, H.-L. Wei and Y.-F. Liu, New J. Chem., 2019, 43, 5706.

3 A. V. Aksenov, D. S. Ovcharov, N. A. Aksenov, D. A. Aksenov, O. N. Nadein and M. Rubin, $R S C A d v$., 2017, 7, 29927; A. V. Aksenov, A. S. Lyakhovnenko, T. S. Perlova and I. V. Aksenova, Chem. Heterocycl. Compd., 2011, 47, 916.

4 D. Canevet, M. Salle, G. Zhang, D. Zhang and D. Zhu, Chem. Commun., 2009, 2245; J. L. Segura and N. Martin, Angew. Chem., Int. Ed., 2001, 40, 1372; N. Martin, Chem. Commun., 2013, 49, 7025.

5 A. Jana, S. Bahring, M. Ishida, S. Goeb, D. Canevet, M. Salle, J. O. Jeppesen and J. L. Sessler, Chem. Soc. Rev., 2018, 47, 5614; Y. Wu, M. Frasconi, W. G. Liu, R. M. Young, W. A. Goddard, M. R. Wasielewski and J. F. Stoddart, J. Am. Chem. Soc., 2020, 142, 11835; L. Tan, Y. Guo, Y. Yang, G. Zhang, D. Zhang, G. Yu, W. Xu and Y. Liu, Chem. Sci., 2012, 3, 2530; J. Wu, N. Dupont, S.-X. Liu, A. Neels, A. Hauser and S. Decurtins, Chem. - Asian J., 2009, 4, 392; K. Herve, S.-X. Liu, O. Cador, S. Golhen, Y. Le Gal, A. Bousseksou, H. Stoeckli-Evans, S. Decurtins and L. Ouahab, Eur. J. Inorg. Chem., 2006, 3498.

6 F. Pop and N. Avarvari, Chem. Commun., 2016, 52, 7906; J. J. Bergkamp, S. Decurtins and S.-X. Liu, Chem. Soc. Rev., 2015, 44, 863; E. J. Rohwer, Y. Geng, M. Akbarimoosavi, L. M. L. Daku, O. Aleveque, E. Levillain, J. Hauser, A. Cannizzo, R. Häner, S. Decurtins, R. J. Stanley, T. Feurer and S. X. Liu, Chem. - Eur. J., 2021, 27, 5399.

7 P. Zhou, U. Aschauer, S. Decurtins, T. Feurer, R. Häner and S. X. Liu, Chem. Commun., 2020, 56, 13421.

8 B. Gao, M. Wang, Y. Cheng, L. Wang, X. Jing and F. Wang, J. Am. Chem. Soc., 2008, 130, 8297; L. Zoephel, V. Enkelmann, R. Rieger and K. Muellen, Org. Lett., 2011, 13, 4506; N. Kulisic, S. More and A. Mateo-Alonso, Chem. Commun., 2011, 47, 514.

9 Z.-H. Wu, Z.-T. Huang, R.-X. Guo, C.-L. Sun, L.-C. Chen, B. Sun, Z.-F. Shi, X. Shao, H. Li and H.-L. Zhang, Angew. Chem., Int. Ed., 2017, 56, 13031.

10 H. Bock, D. Subervie, P. Mathey, A. Pradhan, P. Sarkar, P. Dechambenoit, E. A. Hillard and F. Durola, Org. Lett., 2014, 16, 1546; T. H. El-Assaad, K. N. Parida, M. F. Cesario and D. V. McGrath, Green Chem., 2020, 22, 5966.

11 J. Shao, J. Chang and C. Chi, Org. Biomol. Chem., 2012, 10, 7045.

12 V. Vitske, C. König, O. Hübner, E. Kaifer and H.-J. Himmel, Eur. J. Inorg. Chem., 2010, 115.

13 H.-P. Jia, S.-X. Liu, L. Sanguinet, E. Levillain and S. Decurtins, J. Org. Chem., 2009, 74, 5727; C. Jia, S. Liu, C. Tanner, C. Leiggener, L. Sanguinet, E. Levillain, S. Leutwyler, A. Hauser and S. Decurtins, Chem. Commun., 2006, 1878.

14 C. Jia, S.-X. Liu, C. Tanner, C. Leiggener, A. Neels, L. Sanguinet, E. Levillain, S. Leutwyler, A. Hauser and S. Decurtins, Chem. - Eur. J., 2007, 13, 3804.

15 M. Jaggi, C. Blum, B. S. Marti, S.-X. Liu, S. Leutwyler and S. Decurtins, Org. Lett., 2010, 12, 1344.

16 M. J. Frisch, G. W. Trucks, H. B. Schlegel, G. E. Scuseria, M. A. Robb, J. R. Cheeseman, G. Scalmani, V. Barone, G. A. Petersson, H. Nakatsuji, X. Li, M. Caricato, A. V. Marenich, J. Bloino, B. G. Janesko, R. Gomperts, B. Mennucci, H. P. Hratchian, J. V. Ortiz, A. F. Izmaylov, J. L. Sonnenberg, D. Williams-Young, F. Ding, F. Lipparini, F. Egidi, J. Goings, B. Peng, A. Petrone, T. Henderson, D. Ranasinghe, V. G. Zakrzewski, J. Gao, N. Rega, G. Zheng, W. Liang, M. Hada, M. Ehara, K. Toyota, R. Fukuda, J. Hasegawa, M. Ishida, T. Nakajima, Y. Honda, O. Kitao, H. Nakai, T. Vreven, K. Throssell, J. A. Montgomery Jr., J. E. Peralta, F. Ogliaro, M. J. Bearpark, J. J. Heyd, E. N. Brothers, K. N. Kudin, V. N. Staroverov, T. A. Keith, R. Kobayashi, J. Normand, K. Raghavachari, A. P. Rendell, J. C. Burant, S. S. Iyengar, J. Tomasi, M. Cossi, J. M. Millam, M. Klene, C. Adamo, R. Cammi, J. W. Ochterski, R. L. Martin, K. Morokuma, O. Farkas, J. B. Foresman and D. J. Fox, Gaussian 16 Revision C.01, Gaussian, Inc., Wallingford, CT, 2016.

17 P. J. Skabara and M. Sallé, Beilstein J. Org. Chem., 2015, 11, 1528; J. Sly, P. Kasák, E. Gomar-Nadal, C. Rovira, L. Górriz, P. Thordarson, D. B. Amabilino, A. E. Rowan and R. J. M. Nolte, Chem. Commun., 2005, 1255; E. Gomar-Nadal, L. Mugica, J. Vidal-Gancedo, J. Casado, J. T. L. Navarrete, J. Veciana, C. Rovira and D. B. Amabilino, Macromolecules, 2007, 40, 7521; A. M. Amacher, J. Puigmarti-Luis, Y. Geng, V. Lebedev, V. Laukhin, K. Kraemer, J. Hauser, D. B. Amabilino, S. Decurtins and S.-X. Liu, Chem. Commun., 2015, 51, 15063. 